\title{
Auditory representational momentum: Musical schemata and modularity
}

\author{
TIMOTHY L. HUBBARD \\ Eastern Oregon State College, La Grande, Oregon
}

\author{
(Alliston K. Reid, Sponsor)
}

\begin{abstract}
Memory for the final position or orientation of a moving target is often shifted or displaced in the direction of anticipated motion, but whether this memory shift is produced by a cognitively impenetrable or modular process is less clear. Previous research induced expectations regarding future target behavior by presenting examples of that behavior or by verbally instructing the subject about future target behavior; in this experiment, no induction occurs, and subjects tap previously existing expectations. The subjects are presented with highly schematic ascending sequences of three discrete tones corresponding to the tonic (I), dominant (V), and octave (VIII) of a major scale. The third inducing tone (i.e., the octave) is either flattened slightly, sharpened slightly, or in tune. When the third inducing stimulus is slightly mistuned, the subjects' musical schemata would be predicted to shift their memory for the final pitch closer to a proper tuning. The direction of mistuning is varied so that the direction of schema-driven shift is either consistent or inconsistent with the direction of implied pitch motion, thus allowing examination of whether representational momentum is influenced by schemata effects. When the third inducing tone is flattened, memory shift is upward, but when the third inducing tone is sharpened, memory shift is downward. This pattern supports the claim that representational momentum is cognitively penetrable to top-down influence and thus cannot result from a completely modular process.
\end{abstract}

When people visually perceive a moving target that vanishes without warning, the remembered final position or orientation of the target is typically "displaced" or "shifted" in the direction of anticipated motion. This memory shift is influenced by the velocity (Freyd \& Finke, 1985; Hubbard \& Bharucha, 1988), acceleration (Finke, Freyd, \& Shyi, 1986) and direction (Hubbard, 1990) of the motion of the target, the motion of the context in which the target is embedded (Hubbard, 1993), the duration between the disappearance of the target and the probing of the remembered location (Freyd \& Johnson, 1987), the coherence of the inducing sequence (Freyd $\&$ Finke, 1984), the interaction of the target with other elements in the stimulus context (Bharucha \& Hubbard, 1992; Hubbard, 1992b), the implied friction between the target and other elements in the display (Hubbard, 1992b), and whether target motion is embedded within an event hierarchy (Verfaillie \& d'Ydewalle, 1991). This memory shift has been referred to as representational momentum (e.g., Freyd \& Finke, 1984; Kelly \& Freyd, 1987) and as displacement (e.g., Hubbard, 1990; Hubbard \& Bharucha, 1988).

This research was supported by a National Science Foundation ROA grant to the author, which was affixed to Presidential Young Investigator Award BNS-8451356, previously awarded to Jennifer Freyd, and by research funds from Eastern Oregon State College. The author thanks Jennifer Freyd for the use of the Dynamics Lab at the University of Oregon and for helpful discussions. Correspondence concerning this article should be addressed to T. L. Hubbard, Department of Psychology, Eastern Oregon State College, La Grande, OR 97850.
The extent to which representational momentum is "'modular" or "cognitively impenetrable"' has recently been debated (e.g., Finke \& Freyd, 1989; Ranney, 1989). To qualify as modular, a cognitive process must meet several criteria (see Fodor, 1983, for further discussion of modularity): A modular process must be domain specific, rapid and mandatory in its execution, and informationally encapsulated (i.e., insensitive or impenetrable to the beliefs or expectations of the subject). The ideas of modularity and information encapsulation are also closely related to the notion of cognitive impenetrability (i.e., to be considered impenetrable, a cognitive process must be immune to any effects of a subject's beliefs, knowledge, or goals; see Pylyshyn, 1981, 1984).

Representational momentum initially appears to meet many of the criteria for modularity (see Fodor, 1983) because it is rapid (Freyd \& Johnson, 1987) and mandatory (i.e., immune to error feedback; Finke \& Freyd, 1985). Freyd (1987) has also suggested that representational momentum is informationally encapsulated. A number of findings, however, have recently thrown the modularity of representational momentum into doubt. For example, Verfaillie and d'Ydewalle (1991) reported that representational momentum was obtained if the last inducing stimulus was well within the boundaries of a periodic motion (and motion of the target would have continued in the same direction), but that representational momentum was greatly diminished if the last inducing stimulus occurred at the boundary of a periodic motion (and the motion of the target was about to change direction). Hubbard and 
Bharucha (1988; see also Bharucha \& Hubbard, 1992) reported that the judged vanishing point was displaced backward if a target vanished just prior to or at the moment of bouncing off a barrier, but that the judged vanishing point was displaced forward if the target vanished just after bouncing off a barrier; in all cases, displacement was in the direction of anticipated motion rather than in the direction of current motion. In both the Verfaillie and d'Ydewalle and the Hubbard and Bharucha studies, subjects' expectations about the future behavior of the target influenced the memory shifts, suggesting that displacement is not informationally encapsulated. In an even stronger demonstration, Hubbard (in press) presented subjects with verbal cues specifying whether a target would bounce off or crash through a barrier. The validity of the cues was varied, and invalid cues resulted in less forward displacement than did valid cues.

In previous studies, subjects' expectations concerning target behavior were induced within the laboratory setting while they watched the targets over the course of the experimental trials. A further examination of the possible cognitive penetrability of representational momentum would involve inducing changes in magnitude or direction of memory shift without explicitly instructing the subject about possible target behavior and without changing the direction of implied or anticipated motion. One possible way to do this pits the strength of representational momentum against the strength of subjects' musical schemata. ${ }^{1}$ Previous research has shown that subjects possess schemata for elements of the Western musical scale (Bharucha, 1987; Bharucha \& Stoeckig, 1986; Krumhansl, 1990), so if a highly schematic musical sequence were played to subjects, and the final element of the sequence was slightly out of tune, subjects' schemata ought to shift their memory for the final pitch closer to a proper tuning (i.e., in the process of assimilating the stimulus to the schema, the pitch would be distorted in a schemaconsistent direction). The direction of assimilation can be varied so that assimilation is in a direction that is either consistent or inconsistent with implied pitch motion, and thus either consistent or inconsistent with the direction of shift that a cognitively impenetrable representational momentum should produce. Specifically, if for an ascending series the final pitch is flat, then both representational momentum and schemata should shift the memory for the final pitch higher; if for an ascending series the final pitch is sharp, then an impenetrable representational momentum should shift the memory for the final pitch higher, whereas assimilation to a schema should shift the memory for the final pitch lower.

\section{METHOD}

\section{Subjects}

Twelve subjects were recruited from members of the summer community at the University of Oregon by posting notices on bulletin boards. The subjects were paid a small fee for participating in the experiment.

\footnotetext{
Apparatus

The stimuli were generated by an Apple Macintosh IIcx microcomputer and were presented to subjects over a pair of Pioneer headphones (SE-2)
}

connected directly to the computer. The subjects' responses were recorded by the Macintosh.

\section{Stimuli}

All auditory stimuli consisted of sine waves generated by the Macintosh, and pitch intervals were specified using an equal-tempered tuning scale. An auditory mask consisting of 16 tones, each lasting $125 \mathrm{msec}$, was generated at the beginning of each trial. The pitches of each tone in the mask were randomly determined for each trial. The entire mask lasted $2 \mathrm{sec}$, with no pauses between notes. The stimulus sequence consisted of three inducing tones followed by a probe tone. The first inducing tone was either 500,1000 , or $2000 \mathrm{~Hz}$. The second inducing tone was a perfect fifth ( 700 cents) above the first tone. The third inducing tone was either 1,150 cents above the first tone (flat condition), 1,200 cents above the first tone (in-tune condition), or 1,250 cents above the first tone (sharp condition). Thus, the first inducing tone was the tonic of a major scale, the second inducing tone was the perfect fifth, and the third inducing tone was an octave, in tune, slightly flat, or slightly sharp. ${ }^{2}$ Probe pitches were spaced in 25-cent increments, with the lowest probe pitch 50 cents below the pitch of the third inducing tone and the highest probe pitch 50 cents above the pitch of the third inducing tone. The duration of each of the inducing tones and the interstimulus intervals between the tones was $250 \mathrm{msec}$. There was a total of 360 trials, which each subject received in a different random order.

\section{Procedure}

The subjects were run individually, and they initiated each trial by pressing a designated key. The auditory mask was presented for $2 \mathrm{sec}$ followed by a 1 -sec pause and then by the inducing tones. After the end of the third inducing tone, there was a $250-\mathrm{msec}$ pause, followed by presentation of the probe. The subjects were instructed to respond as quickly but as accurately as possible as soon as the probe began playing, and they responded by pressing either a key marked $S$ (for same) or $D$ (for different). The subjects were given 12 practice trials at the beginning of the session and were given more practice trials if they requested them or appeared confused in any way. Practice trials were drawn randomly from the experimental trials. The entire session took approximately $50 \mathrm{~min}$.

\section{RESULTS}

On each trial, there were five possible pitches that the probe could have had, ranging in 25-cent increments from 50 cents below the pitch of the third inducing tone to 50 cents above the pitch of the third inducing tone. Were subjects responding accurately, we would have observed $0 \%$ same responses on the probes that were $-50,-25,+25$, and +50 cents from the pitch of the third inducing tone and $100 \%$ same responses on the probe that was 0 cents from (i.e., identical to) the pitch of the third inducing tone. Two types of analyses were conducted: analysis using estimates of the degree of memory shift, and direct comparison of the predicted asymmetries in the same responses of probe pitches directly above and below the final pitch. Estimates of the degree of memory shift were determined by calculating the weighted mean (i.e., the sum of the products of the proportions of same responses and test positions divided by the sum of the proportions of test responses).

As shown in Figure 1, the tuning of the inducing sequence significantly affected the direction and magnitude of memory shift $\left[F(2,22)=27.84, M S_{\mathrm{e}}=10.55, p<\right.$ $.0001]$. When the third inducing tone was flattened from a pure octave, a shift in the direction of implied pitch motion was found. Shift magnitude was reduced somewhat when the interval consisted of a pure octave. Importantly, 


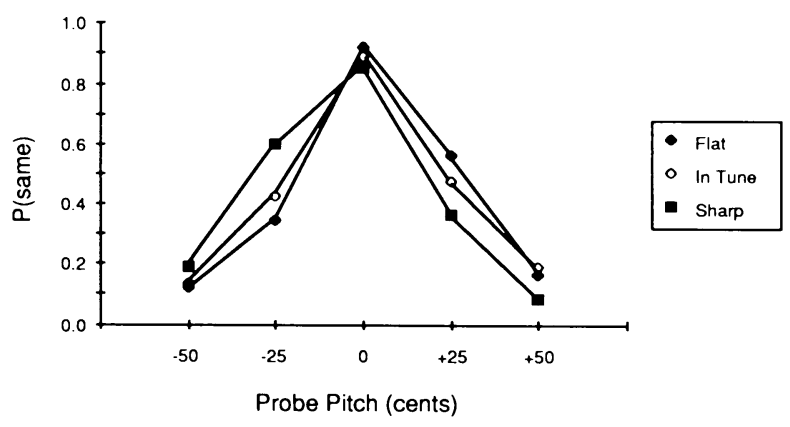

Figure 1. The probability of a same response as a function of probe pitch.

however, the direction of the shift was reversed when the interval spanned by the inducing tones was slightly greater than an octave. Also, $t$ tests (using a Bonferroni correction of $.05 / 3=.017$ ) comparing the probabilities of a same response on the second and fourth probe pitches revealed a higher probability of a same response for a pitch slightly higher than the final pitch in the flat condition $[t(11)=3.50, p=.0025]$ and a higher probability of a same response for a pitch slightly lower than the final pitch in the sharp condition $[t(11)=2.55, p=.013]$. This pattern is consistent with the notion that remembered pitch is displaced toward a true octave interval, as predicted by schema theory, and not in the direction of implied pitch motion, as predicted by theories of representational momentum. The asymmetry in probabilities was not significant in the in-tune condition.

\section{DISCUSSION}

In both the flat and sharp conditions, the directions of the memory shifts are consistent with predictions based on assimilation to a schema suggesting that schematic influences can overwhelm or suppress (or determine) representational momentum. When the final pitch of the inducing sequence was flattened from a true octave, memory for the final pitch was shifted higher, but when the final pitch of the inducing sequence was sharpened from a true octave, memory for the final pitch was shifted lower. Importantly, in both flat and sharp conditions, the direction of implied (and, assumably anticipated) pitch motion was always the same; therefore, the subjects would not have anticipated any change in the direction of target motion. Were this experiment replicated on subjects who had not been immersed in a lifetime of Western tonality, however, effects of octave and fifth schemata might not be seen, and displacement might be primarily in the direction of pitch motion.

Previous work on visual displacement found that subjects' expectations regarding the future behavior (e.g., direction of travel) of a moving target influence the direction and magnitude of displacement. Such expectations would have been recently acquired and probably easily available to subjects' introspections. The type of expectation tapped in this study, however, is different. In this study, the expectation was not initially induced within the experimental setting but was the result of a lifetime of experience within the Western musical milieu. The expectation would not have been recently acquired and would probably not have been easily available to subjects' verbal introspections. Thus, it appears that representational momentum can be modified both by recently acquired expectations (e.g., Hubbard, in press; Hubbard \& Bharucha, 1988) and by long-term schematic expectations. To the extent that these longterm schematic expectations are not easily modifiable, a person's performance would appear to be modular. The pattern produced by the module, however, would itself have been learned.
Memory shifts, then, exhibit aspects of top-down influence. Such topdown influences of expectation are consistent with the claims of Hubbard and Bharucha (1988; Bharucha \& Hubbard, 1992) that displacements cannot be due solely to sensory bottom-up factors such as eye movements. ${ }^{3}$ Even though the direction of the memory shifts differed as a function of the tuning of the third inducing stimulus, however, shifts did still occur, and thus these results are also consistent with the suggestions of Finke and Freyd (1989) that although some aspects of representational momentum may be cognitively penetrable (e.g., the precise path of extrapolation), other aspects are cognitively impenetrable (e.g., the spontaneous rate of extrapolation, the continuity of the extrapolation process, and the inability to halt the extrapolation process). The influences of previously acquired musical schemata on the memory shifts demonstrate that although certain aspects of memory shifts appear to be cognitively impenetrable, other aspects of memory shifts appear to be cognitively penetrable. Because the memory shifts are not completely informationally encapsulated, they cannot result from purely modular processes.

\section{REFERENCES}

HUBBARD, T. L. (1993). The effect of context on visual representational momentum. Memory \& Cognition, 21, 103-114.

HuBbarD, T. L. (in press). Judged displacement: A modular process? the American Journal of Psychology.

HubBard, T. L., \& Bharucha, J. J. (1988). Judged displacement in apparent vertical and horizontal motion. Perception \& Psychophysics, 44, 211-221.

ory induced by implied motions of pattern elements. Journal of Experimental Psychology: Leaming, Memory, \& Cognition, 11, 780-794.

FINKE, R. A., \& FREYD, J. J. (1989). Mental extrapolation and cognitive penetrability: Reply to Ranney and proposals for evaluative criteria. Journal of Experimental Psychology: General, 118, 403-408.

Finke, R. A., FreYd, J. J., \& ShyI, G. C. W. (1986). Implied velocity and acceleration induce transformations of visual memory. Journal of Experimental Psychology: General, 115, 175-188.

FoDor, J. A. (1983). The modularity of mind. Cambridge, MA: MIT Press.

FrEYD, J. J. (1987). Dynamic mental representations. Psychological Review, 94, 427-438.

FREYD, J. J., \& FINKE, R. A. (1984). Representational momentum. Journal of Experimental Psychology: Learning, Memory, \& Cognition, 10, 126-132.

FrEYD, J. J., \& FinKE, R. A. (1985). A velocity effect for representational momentum. Bulletin of the Psychonomic Society, 23, 443-446.

FreYd, J. J., \& Johnson, J. Q. (1987). Probing the time course of representational momentum. Journal of Experimental Psychology: Learning, Memory, \& Cognition, 13, 259-269.

Freyd, J. J., Kelly, M. H., \& DeKay, M. L. (1990). Representational momentum in memory for pitch. Journal of Experimental Psychology: Learning, Memory, \& Cognition, 16, 1107-1117.

Hubbard, T. L. (1990). Cognitive representation of linear motion: Possible direction and gravity effects in judged displacement. Memory \& Cognition, 18, 299-309.

HuBbard, T. L. (1992a). Auditory representational momentum: Surface form, direction, and velocity effects. Manuscript submitted for publication.

HUBBARD, T. L. (1992b). Cognitive representation of motion: Evidence for friction and gravity analogues. Manuscript submitted for publication.

HUBBaRD, T. L. (1993). The effect of context on visual representational momentum. Memory \& Cognition, 21, 103-114.

HuBbarD, T. L. (in press). Judged displacement: A modular process? The American Journal of Psychology.

Hubbard, T. L., \& BharUCha, J. J. (1988). Judged displacement in apparent vertical and horizontal motion. Perception \& Psychophysics, 44, 211-221.

Hubbard, T. L., \& Bharucha, J. J. (1990, November). Is judged dis- 
placement a modular process? Poster presented at the 31st Annual Meeting of the Psychonomic Society, New Orleans.

KELLY, M. H., \& FREYD, J. J. (1987). Explorations of representational momentum. Cognitive Psychology, 19, 369-401.

Krumhansl, C. L. (1990). Cognitive foundations of musical pitch. New York: Oxford University Press.

Pylyshyn, Z. W. (1981). The imagery debate: Analogue media versus tacit knowledge. Psychological Review, 87, 16-45.

PYLYSHYN, Z. W. (1984). Computation and cognition. Cambridge, MA: MIT Press.

RANNEY, M. (1989). Internally represented forces may be cognitively penetrable: Comment on Freyd, Pantzer, and Cheng (1988). Journal of Experimental Psychology: General, 118, 399-402.

Verfaillie, K., \& D'YDEWALle, G. (1991). Representational momentum and event course anticipation in the perception of implied periodical motions. Journal of Experimental Psychology: Learning, Memory, \& Cognition, 17, 302-313.

\section{NOTES}

1. Representational momentum has previously been obtained by using auditory stimuli (e.g., Freyd, Kelly, \& DeKay, 1990; Hubbard, 1992a); therefore, any failures to obtain representational momentum should be due to experimental manipulations and not to modality. Musical stimuli were chosen simply because they offered a strongly schematic stimulus dimension.

2. This particular tone sequence is found in, among other places, Strauss's Thus Spake Zarathustra, and is perhaps more famous as the opening theme from 2001: A Space Odyssey. This particular sequence exploits subjects' tacit knowledge of both the octave and the perfect fifth, which are two of the more basic intervals in Western music.

3. Even so, we certainly would not want to argue that there is not any sensory component to representational momentum; for example, in the collision conditions of Bharucha and Hubbard's (1992) experiments, subjects' eyes may indeed have already been moving in the direction of anticipated motion. In either case, the main point is that a nonsensory factor (e.g., expectations based on previous trials, cues as to the type of forthcoming trial, or schema-based expectations) is controlling the eye movements. Exactly what the auditory equivalent of eye movements would be in the case of auditory representational momentum is, however, not quite clear.

(Manuscript received December 18, 1992.) 